\title{
SHAPE AND CELL WALL SLENDERNESS EFFECTS ON THE STIFFNESS OF WOOD CELL AGGREGATES IN THE TRANSVERSE PLANE
}

\author{
Alp Karakoç, ${ }^{1, \diamond}$
}

\begin{abstract}
The present study investigates a homogenization method in the framework of finite element method to determine the effective stiffness properties of wood cell aggregates in the transverse plane. For this purpose, square and regular hexagonal representative volume elements are chosen to mimic the wood cells. Thereafter, simulation experiments are conducted to understand how different cell shapes and cell wall slenderness, which is cell wall thickness to height ratio, affect the stiffness properties in the transverse plane. The comparison between analytical and computational homogenization results show that square cells have higher elastic moduli than the ones computed for hexagonal cells whereas shear modulus of both cell shapes have more or less the similar values. This can be explained due to the effective deformation mechanisms under different loading conditions. Thus, the present study provides an effective stiffness estimation tool and insight for wood cell aggregates.
\end{abstract}

Keywords: Cell wall shape, computational homogenization, effective stiffness, finite element method, mechanical properties, wood.

\section{INTRODUCTION}

Wood is a natural engineering material which has a microstructure composed of cells. The abundance, moderate durability, favorable strength-to-weight ratio and esthetical concerns make the wood and wood products appealing for different architectural and engineering fields, e.g. structural applications including glulam beams, plywood and particle boards. In these applications, effective stiffness properties in the transverse plane, here referring to radial-tangential plane, are matter of special interest because of the significance of the transverse loads. The effective stiffness properties of wood and wood products can be derived through empirical, analytical and numerical investigations, where the latter two approaches are mainly based on the material microstructure and homogenization.

There have been various empirical studies in the literature to predict the mechanical properties of different wood species, some of which are listed in the following references (Baar et al. 2015, Chauhan and Sethy 2016, Laghdir et al. 2008). Due to long preparation periods of setups and specimens, expensive equipment and need for qualified labor in empirical studies, material homogenization methods, which

${ }^{1}$ Postdoctoral researcher, Department of Forest Products Technology, Aalto University, Espoo, Finland.

•Corresponding author: alp.karakoc@alumni.aalto.fi

Received: 03.09.2015 Accepted: 07.08.2016 
have been the main foci of analytical and numerical investigations, have been introduced and widely implemented (Alkhader and Vural 2008, Charalambakis 2010, Geers et al. 2010). The basic idea behind this phenomenon is to evaluate the effective properties by solving boundary value problem with appropriate boundary conditions defined on a representative volume element RVE, which contains all necessary information (Nemat-Nasser and Hori 1999, Nguyen et al. 2012, Karakoç 2013). Key advantages are to be able to introduce all necessary geometrical and mechanical information related to different scales of the material and use different mechanical models, e.g. beam and plate models, in material sub-scales (Freund et al. 2014, Gibson and Ashby 1999, Wang et al. 2005). Especially, with the introduction of powerful computers in recent years, computational homogenization tools have been widely used for material characterization of complex structures such as wood and wood products, e.g. to determine the effective stiffness properties of cellular materials such as softwood species in the transverse plane (Hofstetter et al. 2009, Karakoç et al. 2013, Rafsanjani 2013, Saavedra Flores 2011).

In order to study the wood cell mechanical behavior, a strain driven computational homogenization model in the framework of finite element method, which couples the geometrical and mechanical description of wood cells with the effective properties of aggregate, is presented. The objectives of the current study are (1) to determine the effective stiffness properties of wood cell aggregates in the transverse plane by using square and regular hexagonal RVEs shown in Figure 1, (2) to understand the effect of cell wall slenderness that is cell wall thickness to height ratio on these effective properties, (3) and to compare the obtained stiffness properties with the analytical results given in the literature on the basis of RVE shape and cell wall slenderness. Therefore, the present study aims at providing an effective stiffness estimation tool and insight for wood cell aggregates.

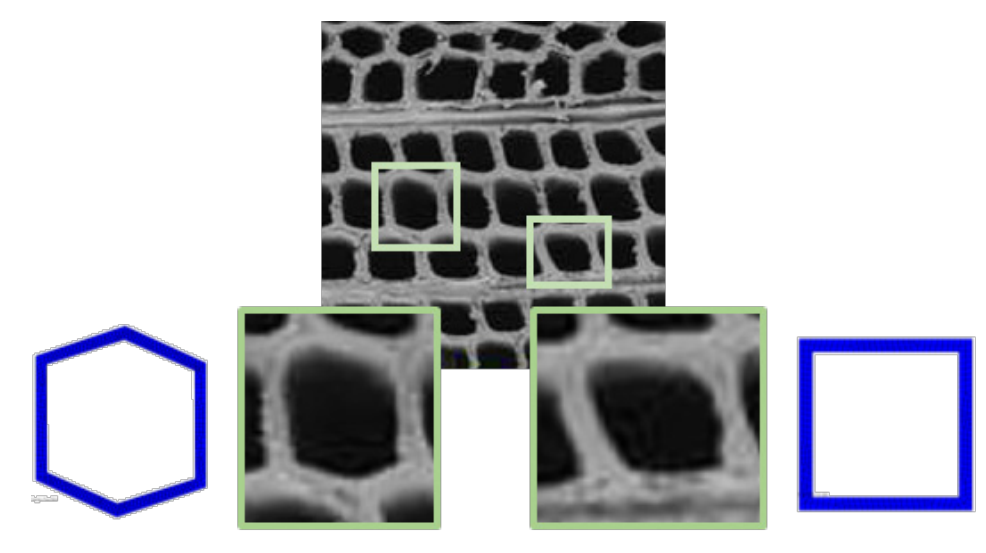

Figure 1. Wood cell aggregate, and hexagonal and square RVEs mimicking the wood cells (edited micrograph, Sjölund et al. 2014).

\section{MATERIALS AND METHODS}

\section{First order strain driven computational homogenization}

In order to determine the effective stiffness properties, first order strain driven computational homogenization can be used. In this method, macroscopic strain $e_{i j}^{\mathrm{M}}$ for $i, j \in\{X, Y\}$ is assumed to be uniform over the material and imposed at the RVE boundary $\partial \omega$ as illustrated in Figure 2. Therefore, first order microscopic displacement field decomposition for RVE can be given as (Rafsanjani 2013) 


$$
\vec{u}^{\mathrm{m}}=\vec{r} \cdot \mathrm{e}^{\mathrm{M}}+\underline{\vec{u}}
$$

for which the first addend on the right hand side represents the macroscopic displacement contribution and second addend represents the displacement fluctuation field $\overrightarrow{\vec{u}}$ due to heterogeneities within the RVE (Geers et al. 2010). Here, $\vec{r}$ represents the position vector with respect to any described origin.

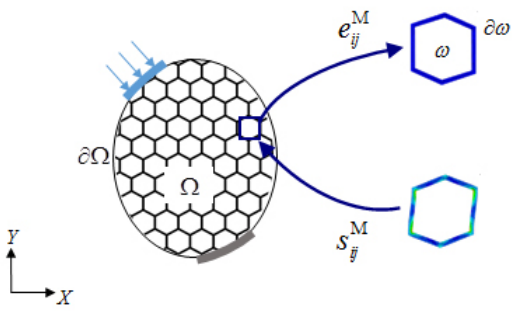

Figure 2. Strain driven homogenization scheme with imposed macroscopic strain $e_{i j}^{\mathrm{M}}$ and computed stress $s_{i j}^{\mathrm{M}}$ for $i, j \in\{X, Y\}$. Here, $\Omega$ and $\partial \Omega$ represents the volume and boundary of aggregate, and $\omega$ and $\partial \omega$ represents the volume and boundary of RVE.

Since the overall body is assumed to be composed of periodic arrays of repeating RVEs, continuity conditions for the displacements and tractions must be satisfied at the boundaries of each neighbouring cells. The first can be satisfied by taking the relative positions of the node sets, e.g. node 1 and node 2 at the boundaries, i.e. surfaces, edges, corners. Hence, Eq.(1) can be expanded as

$$
\vec{u}_{1}^{\mathrm{m}}-\vec{u}_{2}^{\mathrm{m}}=\left(\vec{r}_{1}-\vec{r}_{2}\right) \cdot \mathbf{e}^{\mathrm{M}}
$$

where the displacement fluctuation field $\underline{\vec{u}}$ of Eq.(1) vanishes under the periodicity condition. Eq. (2) forms the basis of displacement boundary condition for the periodic arrays. The traction boundary condition is satisfied with anti-periodicity of traction field in case of existence of traction on the boundaries (Nguyen et al. 2012). However, the current study focuses on the displacement boundary conditions; therefore, only Eq.(2) is studied for the periodic boundary conditions.

In order to establish a consistent relation between different scales, Hill-Mandel principle can be used such that (Pham et al. 2013)

$$
\mathbf{s}^{\mathrm{M}}: \mathbf{e}^{\mathrm{M}}=\frac{1}{\omega} \int_{\omega} \mathbf{s}^{\mathrm{m}}: \mathbf{e}^{\mathrm{m}} \mathrm{d} \omega
$$

for which subscript $m$ represents the microscopic or RVE scale. The symbol (:) denotes the double dot operator and for second order tensors it is defined as 


$$
\mathbf{a}: \mathbf{b}=a_{i j} b_{i j}
$$

By using the Gauss theorem, Eq.(3) can be rewritten at the RVE boundary $\partial \omega$ as

$$
\mathbf{s}^{\mathrm{M}}: \mathbf{e}^{\mathrm{M}}=\frac{1}{\omega} \int_{\partial \omega} \vec{t}^{\mathrm{m}} \cdot \vec{u}^{\mathrm{m}} \mathrm{d} \partial \omega
$$

where $\vec{t}^{\mathrm{m}}$ is the microscopic traction vector at $\partial \omega$. By plugging Eq. (1) into Eq.(5),

$$
\mathbf{s}^{\mathrm{M}}: \mathbf{e}^{\mathrm{M}}=\frac{1}{\omega} \int_{\partial \omega} \vec{t}^{\mathrm{m}} \cdot\left(\vec{r} \cdot \mathbf{e}^{\mathrm{M}}\right) \mathrm{d} \partial \omega+\frac{1}{\omega} \int_{\partial \omega} \vec{t}^{\mathrm{m}} \cdot \underline{\vec{u}} \mathrm{~d} \partial \omega,
$$

which yields to

$$
\mathbf{s}^{\mathrm{M}}: \mathbf{e}^{\mathrm{M}}=\frac{1}{\omega} \int_{\partial \omega}\left(\vec{t}^{\mathrm{m}} \otimes \vec{r}\right) \mathrm{d} \partial \omega: \mathbf{e}^{\mathrm{M}}+\frac{1}{\omega} \int_{\partial \omega} \vec{t}^{\mathrm{m}} \cdot \underline{\vec{u}} \mathrm{~d} \partial \omega
$$

Here, the symbol $\otimes$ denotes the dyadic operator. The second integrand at the right hand side vanishes in case of periodic boundary conditions as elaborated in Eq. . Hence, macroscopic stress $s_{i j}^{\mathrm{M}}$ can be expressed as the volume average of the microscopic stress $s_{i j}^{\mathrm{m}}$ such that

$$
\mathbf{s}^{\mathrm{M}}=\frac{1}{\omega} \int_{\partial \omega}\left(\vec{t}^{\mathrm{m}} \otimes \vec{r}\right) \mathrm{d} \partial \omega=\frac{1}{\omega} \int_{\omega} \mathbf{s}^{\mathrm{m}} \mathrm{d} \omega
$$

in which $\omega$ is the total volume of the RVE.

Thereafter, effective stiffness can be determined by means of the relationship between the macroscopic strain $e_{i j}^{\mathrm{M}}$ and stress $s_{i j}^{\mathrm{M}}$ under the linear elasticity assumption. This relationship can be constructed in terms of macroscopic compliance so that (Karakoç and Freund 2013)

$$
\mathbf{e}^{\mathrm{M}}=\mathrm{C}^{\mathrm{M}}: \mathbf{s}^{\mathrm{M}}
$$


Then, Voigt notation for $\mathbf{C}^{\mathrm{M}}$ can be constructed as

$$
\left[\mathbf{C}^{\mathrm{M}}\right]=\left[\begin{array}{c:c:c}
e_{X X}^{\mathrm{M}, 1} & e_{X X}^{\mathrm{M}, 2} & e_{X X}^{\mathrm{M}, 3} \\
e_{Y Y}^{\mathrm{M}, 1} & e_{Y Y}^{\mathrm{M}, 2} & e_{Y Y}^{\mathrm{M}, 3} \\
2 e_{X Y}^{\mathrm{M}, 1} & 2 e_{X Y}^{\mathrm{M}, 2} & 2 e_{X Y}^{\mathrm{M}, 3}
\end{array}\right]\left[\begin{array}{c:c:c}
s_{X X}^{\mathrm{M}, 1} & s_{X X}^{\mathrm{M}, 2} & s_{X X}^{\mathrm{M}, 3} \\
s_{Y Y}^{\mathrm{M}, 1} & s_{Y Y}^{\mathrm{M}, 2} & s_{Y Y}^{\mathrm{M}, 3} \\
s_{X Y}^{\mathrm{M}, 1} & s_{X Y}^{\mathrm{M}, 2} & s_{X Y}^{\mathrm{M}, 3}
\end{array}\right]^{-1}
$$

for which $\left[\mathbf{C}^{\mathrm{M}}\right]$ is taken to be symmetric matrix. Superscripts 1, 2, 3 represent three different loading conditions fülfilling the minimum requirement to obtain the compliance matrix. Under the assumption of orthotropic material properties

$$
\left[\mathbf{C}^{\mathrm{M}}\right]=\left[\begin{array}{ccc}
1 / E_{X} & -v_{Y X} / E_{Y} & 0 \\
-v_{X Y} / E_{X} & 1 / E_{Y} & 0 \\
0 & 0 & 1 / G_{X Y}
\end{array}\right]
$$

for which $E_{X}, E_{Y}$ are the elastic moduli, $G_{X Y}$ are the shear modulus and $v_{X Y}, v_{Y X}$ are the Poisson's ratios with reciprocal relation $v_{X Y} / E_{X}^{X Y}=v_{Y X} / E_{Y}$ (Laghdir et al. 2008).

\section{Implementation of computational homogenization}

In this study, square and regular hexagonal RVEs are investigated, which has the potential to mimic the nature of wood cells. Therefore, RVEs are first discretized into finite elements and the kinematic boundary condition given in Eq. is applied on matching node sets that form the periodicity conditions. The mapping is exemplified for both RVE geometries in Figure 3.

a)

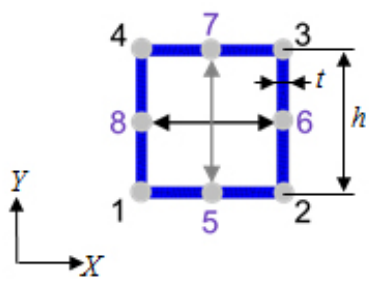

b)

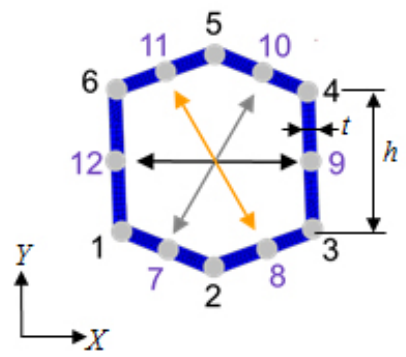

Matching nodes:

Corner nodes: N1 - N2

$\mathrm{N} 1-\mathrm{N} 3$

$\mathrm{N} 1-\mathrm{N} 4$

Edge nodes: N5 - N7

N6 - N8

Matching nodes:

Corner nodes: N1 - N3

N1 - N5

$\mathrm{N} 2-\mathrm{N} 4$

$\mathrm{N} 2$ - N6

Edge nodes: $\quad \mathrm{N} 7-\mathrm{N} 10$

$\mathrm{N} 8-\mathrm{N} 11$

N9 - N12

Figure 3. Geometrical properties and matching nodes of investigated RVEs for periodic boundary conditions: a) 8-noded square RVE, b) 12-noded regular hexagonal RVE. Here, $t$ is cell wall thickness and $h$ is cell wall height. 


\section{RESULTS AND DISCUSSION}

\section{Design of experiments}

In the present study, cell walls are taken to be transversely isotropic under the assumption of uniform distribution of cell wall material in the plane of interest. The detailed information of the assumption and supporting empirical data can be found in the literature (Astley et al. 1998). Hence, mechanical parameters of the cell walls are selected as $E_{\mathrm{s}}=9 \mathrm{GPa}$ and $v_{\mathrm{s}}=0,4$; which refer to cell wall elastic modulus and Poisson's ratio, respectively, as provided in the previous studies (Kahle and Woodhouse 1994). In relation to Eq. , three different in-plane loading conditions, i.e. tensile load along $X$ - and $Y$ - directions and $X Y$-shear load as listed in Table 1 and exemplified in Figure 4, are used to compute the effective stiffness properties and understand the effect of cell wall slenderness $t / h$, for which $t=2 t, t$ is cell wall thickness, and $h$ refers to cell wall height (as depicted in Figure 3 ), on the computed properties. The cell wall slenderness is taken to be in the range so that $\underline{t} / h \in[0,04 ; 0,40]$ with increment of $\Delta \underline{t} / h=0,04$.

Table 1. Loading conditions.

\begin{tabular}{|c|c|c|c|}
\hline \multirow{2}{*}{ Case } & \multicolumn{3}{|c|}{ Imposed strains } \\
\cline { 2 - 4 } & $e_{X X}^{\mathrm{M}}$ & $e_{Y Y}^{\mathrm{M}}$ & $2 e_{X Y}^{\mathrm{M}}$ \\
\hline 1 & 0,01 & 0 & 0 \\
\hline 2 & 0 & 0,01 & 0 \\
\hline 3 & 0 & 0 & 0,01 \\
\hline
\end{tabular}

a)
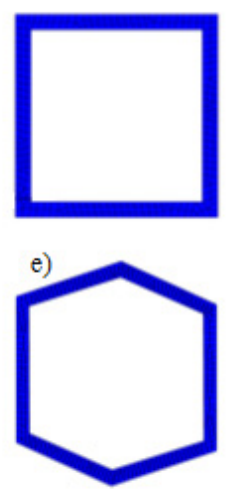

b)

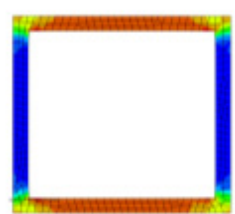

f)

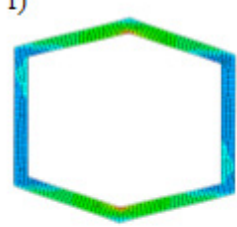

c)

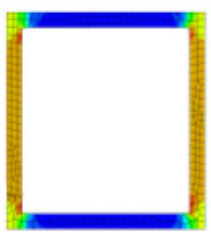

g)

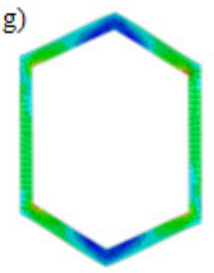

d)

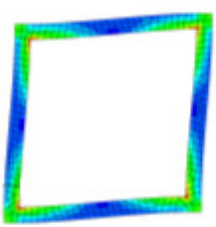

h)

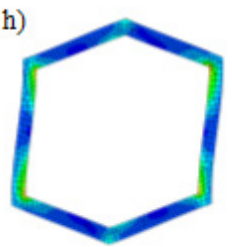

Figure 4. Representation of loading conditions based on the listed cases in Table 1 (presented deformations are scaled larger than the actual ones for readability):a) undeformed square RVE, b) square RVE subjected to $\left.e_{X X}^{\mathrm{M}}, \mathrm{c}\right)$ square RVE subjected to $e_{Y Y}^{\mathrm{M}} \mathrm{d}$ ) square RVE subjected to $e_{X Y}^{\mathrm{M}}$, e) undeformed hexagonal RVE ${ }_{M}$ ) hexagonal RVE subjected to $e_{X X}^{M}{ }_{M}$ ) hexagonal RVE subjected to $\left.e_{Y Y}^{\mathrm{M}}, \mathrm{h}\right)$ hexagonal RVE subjected to $e_{X Y}^{\mathrm{M}}$. 


\section{RESULTS}

For determining the effective stiffness properties of wood cell aggregates derived in Eq. and understanding the effect of cell wall slenderness, simulation experiments are carried out on square and regular hexagonal RVEs in the frameworks of finite element and computational homogenization methods. For this purpose, Abaqus CAE is used and solution domains are discretized into sets of finite elements, e.g. 6-node quadratic triangular CPS6 provided in Abaqus/CAE software. The results are then compared with the analytical results provided in the literature.

\section{Square RVE}

The analytical solutions for cellular RVEs are principally derived considering the cell shape and related deformation mechanisms. For example, in case of square RVEs, cell walls are assumed to behave as a rigid structure than a mechanism (Deshpande et al. 2001). Therefore, stretching is taken to be dominant and bending is neglected under uniaxial loading. However, under shear loading, cell walls are assumed to deform due to bending (Wang et al. 2005). Under these assumptions, previously derived formulae of elastic parameters in the transverse plane are listed in Table 2. Linear relationship between the cell wall slenderness and effective elastic moduli, and cubic relationship between the cell wall slenderness and shear modulus are obvious from this table.

Table 2. Effective elastic moduli, shear modulus and Poisson's ratios as functions of cell wall slenderness $\underline{t} / h$, where $\underline{t}=2 t$ in Survey I and II (Freund et al. 2014, Wang et al. 2005).

\begin{tabular}{|c|c|c|}
\hline & $\begin{array}{c}\text { Survey I } \\
\text { (Freund } \text { et al. 2014) }\end{array}$ & $\begin{array}{c}\text { Survey II } \\
\text { (Wang } \text { et al. 2005) }\end{array}$ \\
\hline$E_{X} / E_{s}$ & $\underline{t} / h$ & $\underline{t} / h$ \\
\hline$E_{Y} / E_{s}$ & $\underline{t} / h$ & $\underline{t} / h$ \\
\hline$G_{X Y} / E_{s}$ & $\frac{1}{2}(\underline{t} / h)^{3}$ & $\frac{1}{2}(\underline{t} / h)^{3}$ \\
\hline$v_{X Y}$ & 0 & $v_{\mathrm{s}}(\underline{t} / h)$ \\
\hline$v_{Y X}$ & 0 & $v_{\mathrm{s}}(\underline{t} / h)$ \\
\hline
\end{tabular}

The comparison graphs presented in Figure 5 also indicate the linear and cubic relations of moduli and $\underline{t} / h$. It can be seen that analytical results for elastic moduli and shear modulus are in accordance with the computational homogenization results. It can be also deduced that there is a positive effect of $\underline{t} / h$ on moduli. On the other hand, Surveys I and II do not estimate the Poisson's ratios correctly that are obtained from the computational homogenization. The main reason can be due to taking only axial deformations into account in the analytical solutions. Similar to effective moduli, $\underline{t} / h$ has a positive 
influence on the Poisson ratio's (Figure 5).

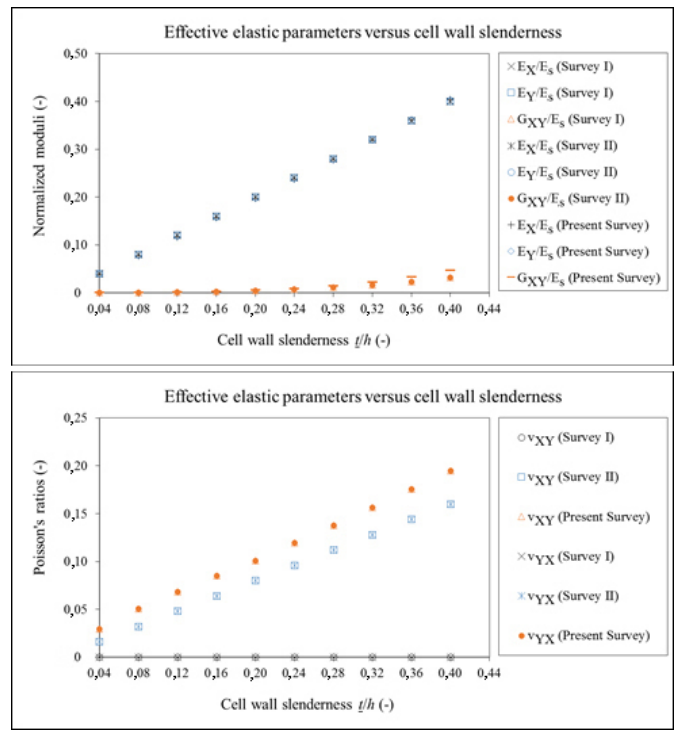

Figure 5. Effective elastic parameters via analytical and computational homogenization results.

\section{Hexagonal RVE}

In case of hexagonal RVEs, cell walls are assumed to behave as mechanism than rigid structure where the bending moments are the dominant deformation mechanisms under both uniaxial and shear loading (Deshpande et al. 2001). Under the assumption, previously derived formulae of elastic parameters in the transverse plane are listed in Table 3. As seen in this table, relationship between the cell wall slenderness and elastic parameters have a more complicated representation compared to the ones for square RVEs. 
Table 3. Effective elastic moduli, shear modulus and Poisson's ratios as functions of cell wall slenderness $\underline{t} / h$, where $\underline{t}=2 t$ in Survey I and III (Freund et al. 2014, Gibson and Ashby 1999).

\begin{tabular}{|c|c|c|}
\hline & $\begin{array}{c}\text { Survey I } \\
\text { (Freund } \text { et al. 2014) }\end{array}$ & $\begin{array}{c}\text { Survey III } \\
\text { (Gibson and Ashby 1999) }\end{array}$ \\
\hline$E_{X} / E_{s}$ & $\frac{4}{\sqrt{3} \frac{(\underline{t} / h)^{3}}{1+3(\underline{t} / h)^{2}}}$ & $\frac{4}{\sqrt{3}} \frac{(\underline{t} / h)^{3}}{1+\left(5,4+1,5 v_{\mathrm{s}}\right)(\underline{t} / h)^{2}}$ \\
\hline$E_{Y} / E_{s}$ & $\frac{4}{\sqrt{3} \frac{(\underline{t} / h)^{3}}{1+3(\underline{t} / h)^{2}}}$ & $\frac{4}{\sqrt{3}} \frac{(\underline{t} / h)^{3}}{1+\left(5,4+1,5 v_{\mathrm{s}}\right)(\underline{t} / h)^{2}}$ \\
\hline$G_{X Y} / E_{s}$ & $\frac{1}{\sqrt{3} \frac{(\underline{t} / h)^{3}}{1+(\underline{t} / h)^{2}}}$ & $\frac{1}{\sqrt{3} \frac{(\underline{t} / h)^{3}}{1+\left(3,3+1,75 v_{\mathrm{s}}\right)(\underline{t} / h)^{2}}}$ \\
\hline$v_{X Y}$ & $\frac{1-(\underline{t} / h)^{2}}{1+3(\underline{t} / h)^{2}}$ & $\frac{1+\left(1,4+1,5 v_{\mathrm{s}}\right)(\underline{t} / h)^{2}}{1+\left(5,4+1,5 v_{\mathrm{s}}\right)(\underline{t} / h)^{2}}$ \\
\hline$v_{Y X}$ & $\frac{1-(\underline{t} / h)^{2}}{1+3(\underline{t} / h)^{2}}$ & $\frac{1+\left(1,4+1,5 v_{\mathrm{s}}\right)(\underline{t} / h)^{2}}{1+\left(5,4+1,5 v_{\mathrm{s}}\right)(\underline{t} / h)^{2}}$ \\
\hline
\end{tabular}

The comparison graphs presented in Figure 6 indicate that there is a positive effect of $\underline{t} / h$ on the effective moduli. However, with increase in $t / h$, there is a decrease in the Poisson's ratios. In addition to these, it is also observed that Surveys I and III are in accordance with the computational homogenization results especially for low values of $\underline{t} / h$, e.g. $\underline{t} / h \square 0,25$. This is a valuable outcome, e.g for softwood cells which usually have the cell wall slenderness values in this $\underline{t} / h$ range. 


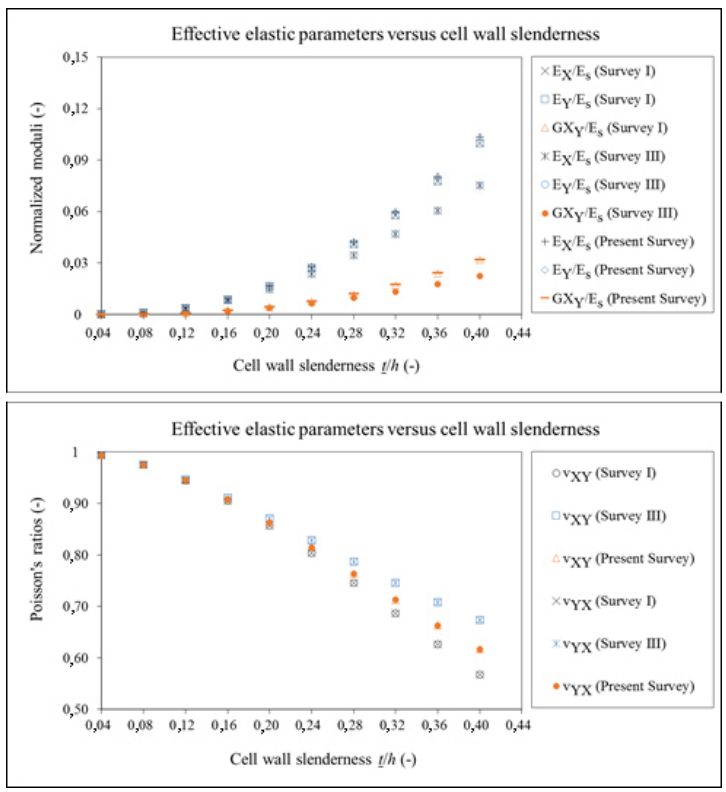

Figure 6. Comparison of effective elastic parameters based on analytical and computational homogenization results.

Another interesting outcome is that elastic moduli of hexagonal RVEs is lower than the one for square RVEs, which is due to the dominant effect of stretching over bending. Moreover, shear modulus have more or less the similar values due to bending as the deformation mechanism for both types of RVEs.

\section{CONCLUSIONS}

In the present study, effective stiffness properties of wood cell aggregates, which are composed of either square or regular hexagonal RVEs, are investigated by means of analytical and computational homogenization methods. The analytical formulae are derived considering the cell shape and related deformation mechanisms, i.e. bending and stretching in the current study. For the computational homogenization, RVEs are first discretized into finite elements and kinematic boundary equations are applied to the matching nodes so as to generate periodic boundary conditions, which is commonly used in multiscale modelling of cellular materials. The simulations are carried by using the linear elastic constitutive equations and effective stiffness properties are obtained by means of the relationship between the macroscopic strain $e_{i j}^{\mathrm{M}}$ and stress $S_{i j}^{\mathrm{M}}$.

The homogenization results show that square RVEs have higher elastic moduli values compared to regular hexagonal RVEs due to the dominancy of stretching over bending, which has been briefly explained here and elaborated in the literature. However, there are slight differences for the shear moduli of both RVE types, which can be explained with the dominant effect of bending under shear loading. The investigated shapes are common for many types of softwood species. Therefore, both the analytical and computational homogenization results in the tables and comparison graphs work as an effective stiffness estimation tool in case of knowing the mechanical and geometrical properties of wood cells. Hence, the use of present methodology is expected to decrease the operational cost and time and advances the state-of-the-art in the field of wood and cellular materials mechanics. 


\section{ACKNOWLEDGEMENTS}

The author gratefully acknowledge the financial supports of Aalto University-PuuPostdoc research fund and Tekniikan edistämissäätiö TES through Foundations' Post Doc Pool, Finland, and technical support of CSC Finland.

\section{REFERENCES}

Alkhader, M.; Vural, M. 2008. Mechanical response of cellular solids: Role of cellular topology and microstructural irregularity. Int J Eng Sci 46:1035-1051.

Astley, R.J.; Stol, K.A.; Harrington, J.J. 1998. Modelling the elastic properties of softwood. Holz als Roh- und Werkstoff 56:43-50.

Baar, J.; Tippner, J.; Rademacher, P. 2015. Prediction of mechanical properties - modulus of rupture and modulus of elasticity - of five tropical species by nondestructive methods. Maderas-Cienc Tecnol 17: 239-252.

Charalambakis, N. 2010. Homogenization techniques and micromechanics. A survey and perspectives. Applied Mechanics Review 63:030803-1-10.

Chauhan, S.; Sethy, A. 2016. Differences in dynamic modulus of elasticity determined by three vibration methods and their relationship with static modulus of elasticity. Maderas-Cienc Tecnol 18:373-382.

Deshpande, V.S.; Ashby, M.F.; Fleck, N.A. 2001. Foam topology: bending versus stretching dominated architectures. Acta Materialia 49:1035-1040.

Freund, J.; Karakoç, A.; Sjölund, J. 2014. Computational homogenization of regular cellular material according to classical elasticity. Mech Mater 78:56-65.

Geers, M.G.D.; Kouznetsova, V.G.; Brekelmans, W.A.M. 2010. Multi-scale computational homogenization: Trends and challenges. J Comput Appl Math 234:2175-2182.

Gibson, L.; Ashby, M. 1999. Cellular Solids: Structure and Properties. Cambridge University Press, Cambridge.

Hofstetter, K.; Eitelberger, J.; Bader, T.K.; Hellmich, Ch.; Eberhardsteiner, J. 2009. Computational Multiscale Approach to the Mechanical Behavior and Transport Behavior of Wood. Computational Structural Engineering: Proceedings of the International Symposium on Computational Structural Engineering: 79-85.

Kahle, E.; Woodhouse, J. 1994. The influence of cell geometry on elasticity of softwood. Journal of Materials Science 29:1250-1259.

Karakoç, A. 2013. Effective stiffness and strength properties of cellular materials in the transverse plane. Doctoral thesis, Aalto University, Espoo, Finland.

Karakoç, A.; Freund, J. 2013. A direct simulation method for the effective in-plane stiffness of cellular materials. International Journal of Applied Mechanics 5(3). Number of article: 1350034. 
Karakoç, A.; Santaoja, K.; Freund, J. 2013. Simulation experiments on the effective in-plane compliance of the honeycomb materials. Composite Structures 96:312-320.

Laghdir, A.; Fortin, Y.; De la Cruz, C.M.; Hernandez, R.E. 2008. Development of a technique to determine the 3D elasticity tensor of wood as applied to drying stress modeling. Maderas-Cienc Tecnol 10(1):35-44.

Nemat-Nasser, S.; Hori, M. 1999. Micromechanics: Overall Properties of Heterogeneous Materials. North Holland, Amsterdam.

Nguyen, V.; Béchet, E.; Geuzaine, C.; Noels, L. 2012. Imposing periodic boundary condition on arbitrary meshes by polynomial interpolation. Computational Materials Science 55:390-406.

Pham, K.; Kouznetsova, V.G.; Geers, M.G.D. 2013. Transient computational homogenization for heterogeneous materials under dynamic excitation. J Mech Phys Solids 61:2125-2146.

Rafsanjani, A. 2013. Multiscale poroelastic model: bridging the gap from cellular to macroscopic scale. ETH Zurich, Zurich, Switzerland.

Saavedra-Flores, E.I.; Murugan, M.S.; Friswell, M.I.; de Souza-Neto, E.A. 2011. Computational multi-scale constitutive model for wood cell wall and its application to the design of bio-inspired composites. Proc. SPIE 7975, Bioinspiration, Biomimetics, and Bioreplication 7975.

Sjölund, J.; Karakoç, A.; Freund, J. 2014. Accuracy of regular wood cell structure model. Mech Mater 76:35-44.

Wang, A.J.; Kumar, R.S.; McDowell, D.L. 2005. Mechanical Behavior of Extruded Prismatic Cellular Metals. Mechanics of Advanced Materials and Structures 12:185-200. 